\title{
Anomalous resistivity and the electron-polaron effect in the two-band Hubbard model with one narrow band
}

\author{
M. Yu. Kagan ${ }^{1}$, V.V. Val'kov \\ ${ }^{1}$ P.L. Kapitza Institute for Physical Problems, Kosygina st. 2, 119334 Moscow, Russia, \\ kagan@kapitza.ras.ru \\ ${ }^{2}$ Kirenskii Institute of Physics, Akademgorodok 50, building 38, 660036 Krasnoyarsk, Russia, \\ vvv@iph.krasn.ru
}

\begin{abstract}
We search for anomalous normal and superconductive behavior in the two-band Hubbard model with one narrow band. We analyze the influence of electron-polaron effect and Altshuler-Aronov effect on effective mass enhancement and scattering times of heavy and light components in the clean case. We find anomalous behavior of resistivity at high temperatures $T>W_{h}^{*}$ both in 3D and 2D situation. The SC instability in the model is governed by enhanced Kohn-Luttinger effect for p-wave pairing of heavy electrons via polarization of light electrons.
\end{abstract}

Key words: electron-polaron effect, two-band Hubbard model, marginality, anomalous resistivity

\section{Introduction.}

The two-band model plays an important role both in the physics of conventional multiband s-wave superconductors like $\mathrm{Nb}[1,2]$ and in the physics of high- $\mathrm{T}_{\mathrm{c}}$ materials [3]. It can be useful also for the description of anomalous normal and superconducting properties in different unconventional superconductors such as ruthenates $\mathrm{Sr}_{2} \mathrm{RuO}_{4}, \mathrm{MgB}_{2}$, new superconductors (SC) based on FeAs layers such as $\mathrm{BaFe}_{2}\left(\mathrm{As}_{1-\mathrm{x}} \mathrm{P}_{\mathrm{x}}\right)_{2}$ [4,5], layered semimetals, dichalcogenites and superlattices, organic superconductors et al. [6-8]. At the recent conference on stripes and high Tc superconductivity in Rome the fermion-boson multiband SC and Bose-BCS crossover was also analyzed in $[9,10]$. In this context we could mention as well $[11,12]$. In the present paper we consider two-band fermionic Hubbard model with one narrow band [13,14]. This model is a very rich one. It describes adequately mixed valence systems such as uranium-based HF and possibly also some other novel superconductors and transition-metal systems with orbital degeneracy such as complex magnetic oxides in optimally doped case. Moreover it contains such highly nontrivial effect as Electron Polaron Effect $[15,16]$ in the homogeneous state. Let us verify this model with respect to marginality [17-19] and anomalous resistivity characteristics.

\section{Two-band Hubbard model.}

In real space the Hamiltonian of the two-band Hubbard model reads:

$$
\hat{H}^{\prime}=-t_{h} \sum_{<i j>\sigma} a_{i \sigma}^{+} a_{j \sigma}-t_{L} \sum_{<i j>\sigma} b_{i \sigma}^{+} b_{j \sigma}-\varepsilon_{0} \sum_{i \sigma} n_{i h}^{\sigma}-\mu \sum_{i \sigma}\left(n_{i \sigma}^{L}+n_{i \sigma}^{h}\right)+U_{h h} \sum_{i} n_{i h}^{\uparrow} n_{i h}^{\downarrow}+U_{L L} \sum_{i} n_{i L}^{\uparrow} n_{i L}^{\downarrow}+\frac{U_{h L}}{2} \sum_{i} n_{i L} n_{i h}
$$


We consider low density $\left(n_{h}+n_{L}\right) d^{D} \ll<$ and strong-coupling limit $U_{h h} \sim U_{h L} \sim U_{L L}>W_{L}>W_{h}$ of this model, $\mu$ is chemical potential. The band structure is presented in Fig. 1.

\subsection{Electron-polaron effect.}

We call the electron-polaron effect (EPE) the non-adiabatical part of many-particle wave function which describes the heavy particle dressed in a cloud of virtual electron-hole pairs of light particles. Nonadiabaticity of the cloud in some energy interval manifests itself when the heavy particle moves from one elementary cell to a neighboring one. Formally EPE is connected with interband Hubbard interaction $U_{h L}$. In the second order of perturbation theory

$m_{h}^{*} / m_{h}=Z_{\mathrm{h}}^{-1}=1+b \ln \frac{m_{h}}{m_{L}}$

where $b=2 f_{0}^{2}, \mathrm{Z}_{\mathrm{h}}^{-1}=1-\left.\frac{\partial \Sigma_{\mathrm{hL}}\left(\omega, \varepsilon_{q}\right)}{\partial \omega}\right|_{\omega \rightarrow 0}$

$Z_{h}$ is Z-factor of heavy particle. In Born approximation $f_{0}=U_{h L} \nu_{L}\left(\varepsilon_{F}\right)$. In more general case of low density and strong Hubbard interaction

$\mathrm{U}_{\mathrm{hL}}>\mathrm{W}_{\mathrm{L}}: f_{0}=\frac{2 d p_{F}}{\pi}$

Galitskii gas parameter in 3D, $d$ is intersite distance. In 2D

$$
f_{0}=\frac{1}{2 \ln 1 / d p_{F}}
$$

- gas-parameter of Bloom. Generally (after collecting of the polaron exponent) the effective mass reads $\frac{m_{h}^{*}}{m_{h}} \sim\left(\frac{m_{h}}{m_{L}}\right)^{b /(1-b)}[15,16]$. In the unitary limit the polaron exponent $b$ can reach the value of $1 / 2$ and thus $\frac{m_{h}^{*}}{m_{h}} \sim \frac{m_{h}}{m_{L}}$. Accordingly $\frac{m_{h}^{*}}{m_{L}} \sim\left(\frac{m_{h}}{m_{L}}\right)^{2}$ and if we start with $\frac{m_{h}}{m_{L}} \sim 10$ in LD approximation for example we can finish with $\frac{m_{h}^{*}}{m_{L}} \sim 100$ due to many-body effects. Thus EPE can possibly explain the origin of a heavy mass in uranium-based HF.

\subsection{Tendency towards phase separation.}

Let us consider other mechanisms of mass-enhancement. The EPE is connected with Z-factor of heavy particle

(with $\left.\left.\frac{\partial \Sigma_{h L}\left(\omega, \varepsilon_{\vec{q}}\right)}{\partial \omega}\right|_{\omega \rightarrow 0}\right)$ 
However in 3D-case momentum dependence of heavy-light self energy

$$
\left.\frac{\partial \Sigma_{h L}\left(\omega, \varepsilon_{\vec{q}}\right)}{\partial \varepsilon_{\vec{q}}}\right|_{q \rightarrow p_{F}}
$$

also becomes very important. Hence as it is shown in $[13,14]$ the full expression for $m^{*}{ }_{h} / m_{h}$ in the second order of perturbation theory reads

$$
\frac{m_{h}^{*}}{m_{h}}=1+b \ln \frac{m_{h}}{m_{L}}+\frac{b}{18} \frac{m_{h} n_{h}}{m_{L} n_{L}}
$$

and possess the additional term which is linear in the bare mass-ration $m_{h} / m_{L}$. If in LD approximation $m_{h} \sim 10 m_{L}$, then this term becomes dominant over EPE contribution $\sim \ln \frac{m_{h}}{m_{L}}$ for large density mismatch $n_{h} \geq 5 n_{L}$. It is very interesting that in $3 \mathrm{D}$ the same parameter $b \frac{m_{h} n_{h}}{m_{L} n_{L}} \geq 1$ governs the tendency towards phase-separation in the two-band model yielding negative partial compressibility

$$
\chi_{h h}^{-1} \sim c_{h}^{2} \sim\left(n_{h} / m_{h}\right)\left(\partial \mu_{h} / \partial n_{h}\right)
$$

where $\mu_{h}$ is chemical potential of the heavy particle. This result is in qualitative agreement with predictions of mean-field type variational analysis [20]. Note that in 2D case the contribution to $m_{h}{ }_{h}$ from $\frac{\partial \Sigma_{h L}}{\partial \varepsilon_{\vec{q}}}$ and the tendency towards phase-separation are absent due to specific form of polarization operator $[13,14]$.

\section{Transport properties}

\subsection{Resistivity in the homogeneous case in 3D.}

Exact solution of coupled kinetic equations with an account of umklapp processes yields for $p_{F h} \sim p_{F L} \sim p_{F} \sim 1 / d$ and low temperature $T<W_{h}^{*}<W_{L}$ for the inverse scattering times: $1 / \tau_{L} \sim 1 / \tau_{L h} \sim f_{0}^{2} \frac{T^{2}}{W_{h}^{*}} \frac{m_{h}}{m_{L}} ; 1 / \tau_{h} \sim 1 / \tau_{h L} \sim f_{0}^{2} \frac{T^{2}}{W_{h}^{*}}$. This behavior corresponds to Landau Fermiliquid picture. Accordingly for the conductivities we have: $\sigma_{h} \sim \sigma_{h L} \sim \sigma_{L} \sim \sigma_{L h} \sim \frac{\sigma_{\min }}{b}\left(\frac{W_{h}^{*}}{T}\right)^{2}$ at low temperatures $T<W_{h}^{*}$. Thus the resistivity

$$
\mathrm{R} \sim \frac{1}{\left(\sigma_{h}+\sigma_{L}\right)} \sim \frac{b}{\sigma_{\min }}\left(\frac{T}{W_{h}^{*}}\right)^{2}
$$

where $\sigma_{\min }=\frac{e^{2} p_{F}}{\hbar}$ is minimal Mott-Regel conductivity in 3D. At high temperatures $T>W_{h}^{*}$ the inverse scattering times read: $1 / \tau_{L} \sim 1 / \tau_{L h} \sim b W_{L}, 1 / \tau_{h} \sim 1 / \tau_{h L} \sim b T$. 
Thus heavy component is marginal - heavy electrons more diffusively in the surrounding of light electrons. However, light electrons scatter on the heavy ones as if on a static impurity and thus light component is non-marginal. Correspondingly for the conductivities:

$$
\begin{aligned}
& \sigma_{L} \sim \sigma_{L h} \sim \frac{n_{L} e^{2}}{m_{L}} \tau_{L h} \sim \frac{n_{L} e^{2}}{m_{L} b W_{L}} \sim \frac{\sigma_{\min }}{b}, \\
& \sigma_{h} \sim \sigma_{h L} \sim \frac{\sigma_{\min }}{b}\left(\frac{W_{h}^{*}}{T}\right)^{2}-
\end{aligned}
$$

with an account for Einstein relation $\frac{\partial n_{h}}{\partial \mathcal{E}} \sim \frac{W_{h}^{*}}{T}$ at high temperatures $T>W_{h}^{*}$. Hence the resistivity

$$
\mathrm{R} \sim \frac{1}{\left(\sigma_{L h}+\sigma_{h L}\right)} \sim \frac{b}{\sigma_{\min }\left[1+\left(\frac{W_{h}^{*}}{T}\right)^{2}\right]}-
$$

goes on saturation in 3D case (see Fig.2). This behavior of $R(T)$ is typical for some uranium-based HFcompounds like $\mathrm{UNi}_{2} \mathrm{Al}_{3}$

\subsection{Altshuler-Aronov effect in $2 D$.}

In $2 \mathrm{D}$ case we should take into account weak-localization corrections due to quantum-mechanical backward scattering to classical Drude formulae for conductivity of the light band[21,22]: $\Delta \sigma_{L} / \sigma_{0 L} \sim b \ln \frac{\tau_{\varphi}}{\tau}$, where $\sigma_{0 L}=\frac{\sigma_{\min }}{b}$ is classical Drude conductivity of light band, $\sigma_{\min }=\frac{e^{2}}{\hbar}$ Mott-Regel minimal conductivity in $2 \mathrm{D}, \tau_{\varphi}=\tau_{e e}=\tilde{\tau}_{L L}$ - is decoherence time for light electrons, $\tau=\tau_{e i}=\tau_{L h}$ and $l_{e l}=v_{F L} \tau_{L h}$ are elastic time and length, $L_{\phi}=\sqrt{D \tau_{\varphi}}=v_{F L} \sqrt{\tau_{L h} \tilde{\tau}_{L L}}$ - is diffusive length, and $1 / \tau_{L h} \sim b W_{L}$ as in 3D. Correspondingly $1 / \tilde{\tau}_{L L}=b^{2} T$ - Altshuler-Aronov effect in "dirty" metal in 2D (electron-electron scattering time becomes marginal in dirty limit when between two subsequent scattering events for light electrons, a light electron scatters a lot of time on heavy electrons as if on almost elastic impurities, see Fig.3). Hence the conductivity of the light band: $\sigma_{L}=\frac{\sigma_{\min }}{b}\left(1-b \ln \frac{W_{L}}{b T}\right)$. Thus in $2 \mathrm{D}$ case light component has a tendency towards localization for $b T \geq W_{h}^{*}$. Moreover the additional narrowing of the heavy band and additional localization of the light band are governed for $b T \sim W_{h}^{*}$ by the same parameter $b \ln \left(m_{h} / m_{L}\right) \geq 1$.

\subsection{Resistivity in homogeneous case in 2D.}

Thus instead of desired marginal Fermi-liquid behavior at high-temperatures $T>W_{h}^{*}$ in $2 \mathrm{D}$ we have even more interesting behavior of resistivity $R \sim \frac{1}{\left(\sigma_{L}+\sigma_{h}\right)}$, where $\sigma_{h} \sim \frac{\sigma_{\min }}{b}\left(\frac{W_{h}^{*}}{T}\right)^{2}$ as in the 3D 
case. Namely $R(T)$ in 2D has a maximum and then a localization tail at higher temperatures (see Fig.4). Such resistivity characteristics resembles the curve for $R(T)$ in optimally doped layred CMR-systems.

\subsection{Superconductivity in the two - band model with one narrow band.}

In the homogeneous state the leading instability in the two band model at low electron densities corresponds to p-wave pairing via enhanced Kohn-Luttinger mechanism of SC [23-27]. Namely SC critical temperature is mostly governed by the pairing of heavy electrons via polarization of light electrons. P-wave critical temperature $T_{C 1}$ is strongly dependent upon relative fillings of the two bands $\mathrm{n}_{\mathrm{h}} / \mathrm{n}_{\mathrm{L}}$ and has a large and broad maximum for $n_{h^{\prime}} n_{L^{\sim 4}}$ in 2D [13,14,26,27]. For $\varepsilon_{F h^{\sim(30-50) K}-\text { typical }}$ for HF-compounds or semimetals (superlattices, heterostructures in 2D) $T_{C 1}$ can reach (1-5)K which is quite nice $[13,14]$. The two SC gaps for heavy and light electrons are opened simultaneously below this temperature [6].

\section{Conclusions.}

We have analyzed EPE and other mechanisms of mass-enhancement for the heavy electrons in the framework of the two-band Hubbard model with one narrow band. These mechanisms can produce the effective heavy masses $m_{h}^{*} \sim 100 m_{e}$ which are typical for uranium-based HF-compounds. For a large mismatch between the densities of heavy and light bands $n_{h} \gg n_{L}$ we also found a tendency towards phase-separation in 3D. We evaluate scattering times and resistivities in the homogeneous case in 3D and in 2D. Both in 3D and 2D cases at low temperatures $T<W_{h}^{*}$ the resistivity behaves in Landau Fermiliquid fashion. At high temperatures $T>W_{h}^{*}$ the resistivity in $3 \mathrm{D}$ goes on saturation as in $\mathrm{UNi}_{2} \mathrm{Al}_{3}$. In 2D case due to weak-localization corrections of Altshuler-Aronov type the resistivity has a maximum and then a localization tail at higher temperatures. We analyzed the possibility of SC-transition in this model. The leading instability is towards triplet p-wave pairing and is governed by enhanced KLmechanism of SC for pairing of heavy electrons via polarization of light electrons.

Acknowledgements. We are grateful to P. Fulde, Yu. Kagan, K.I. Kugel, N.V. Prokof'ev, P. Nozieres, and C.M. Varma for the numerous stimulating discussions. We acknowledge financial support of the RFBR grant № 11-02-00741.

\section{References.}

1. Suhl, H., Matthias, T.B., Walker, L.R.: Phys. Rev. Lett. 3, 552 (1959).

2. Geilikman, B.T.: Sov. Phys. Uspekhi 109, 65 (1973)

3. Emery, V.J.: Phys. Rev. Lett. 58, 2794 (1987)

4. Izumov, Yu.A., Kurmaev, E.Z.: High-Tc superconductors on the basis of FeAs-compounds [in Russian], 312 pages, R\&C Dynamics, Moscow (2009);

5. Chubukov, A.V.: plenary talk on Stripes-XI conference in Rome. 10-17 July 2011

6. Baranov, M.A., Kagan, M.Yu.: Sov. Phys. JETP 102, 313 (1991); 
7. Kagan, M.Yu.: Phys. Lett A 152, 303 (1991)

8. Kagan, M.Yu., Efremov, D.V. et al.: JETP Lett 93, 725 (2011)

9. Innocenti, D., Poccia, N., Ricci, A., Valletta, A., Caprara, S., Perali, A., Bianconi, A.:. Phys. Rev. B 82, 184528 (2010)

10. Poccia, N., Fratini, M., Ricci, A., Campi, G., Barba, L., Vittorini-Orgeas, A., Bianconi, G., Aeppli, G., Bianconi, A.: Nature Materials 10, 733 (2011).

11. Combescot, R., Leyronas, X. and Kagan, M.Yu.: Phys. Rev. A 73, 023618 (2006)

12. Menushenkov A.P., Klementev K.V., Kuznetsov A.V., Kagan M.Yu.: Sov. Phys. JETP 93, 615 (2001)

13. Kagan, M.Yu., Val'kov, V.V.: Sov. Phys. Low Temp. 37, 84(2011) and also in "A Lifetime in Magnetism and Superconductivity: A Tribute to Professor David Schoenberg”, Cambridge Scientific Publishers (2011)

14. Kagan, M.Yu., Val'kov, V.V.: Sov. Phys. JETP 113, 156 (2011)

15. Kagan, Yu. and Prokof'ev, N.V.: Sov. Phys. JETP 66, 211 (1987)

16. Kagan, Yu. and Prokof'ev, N.V.: Sov. Phys. JETP 63, 1276 (1986)

17. Varma, C.M. , Littlewood, P.B., Schmitt-Rink, S., Abrahams, E., and Ruckenstein, A.E.: Phys. Rev. Lett. 63, 1996 (1989)

18. Kagan, M.Yu., Val'kov, V.V., Woelfle, P.: Sov. Phys. Low. Temp 37, 1046(2011)

19. Baranov M.A., Kagan M.Yu., Mar'enko M. S.: JETP Lett 58, 709(1993)

20. Kugel, K.I., Rakhmanov, A.L. and Sboychakov, A.O.: Phys. Rev. B 76, 195113 (2007)

21. Altshuler, B.L. and Aronov, A.G. in Modern Problems in Condensed Matter Sciences, v. 10, p.1, Electronelectron interaction in disordered systems, Ed. by A.L. Efros and M. Pollack (North Holland, Amsterdam, The Netherlands, 1985)

22. Kagan, M.Yu. and Aronov, A.G.: Czech. J. Phys. 46, 2061(1996) Proceedings of the $21^{\text {st }}$ International Conference on Low Temperature Physics (LT-21), Prague, The Czech Republic, August 8-14, 1996.

23. Kohn, W., and Luttinger, J.M.: Phys. Rev. Lett. 15, 524(1965)

24. Fay, D. and Layzer, A.: Phys. Rev. Lett. 20, 187 (1968)

25. Kagan, M.Yu.. Chubukov, A.V.: JETP Lett. 47, 525 (1988)

26. Kagan, M.Yu., Chubukov, A.V.: JETP Lett. 50, 517 (1989)

27. Baranov, M.A., Chubukov, A.V. and Kagan, M.Yu.: Int. Jour. Mod. Phys. B. 6, 2471 (1992) 


\section{Figure Captions}

Fig. 1 The band structure in the two-band model with one narrow band. Wh and WL are the bandwidths of heavy and light electrons

Fig. 2 The resistivity characteristics $R(T)$ in the two-band model in 3D.

Fig. 3 Multiple scattering of light particle on the heavy ones in between of the scattering of light particle on another light particle. $L \varphi$ is a diffusive length, 1 is elastic length

Fig. 4 Resistivity R(T) in a 2D case for the two-band model with one narrow band 


\section{FIGURES}

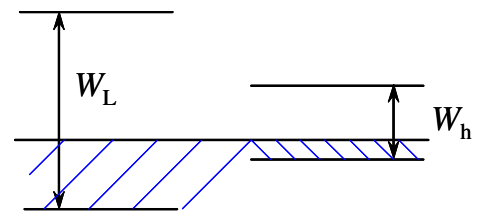

Fig. 1

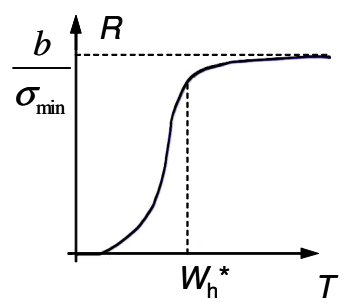

Fig. 2

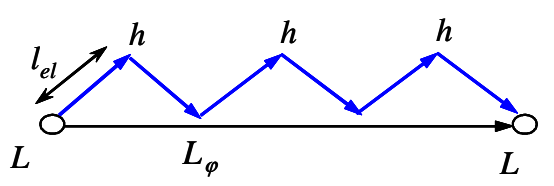

Fig. 3

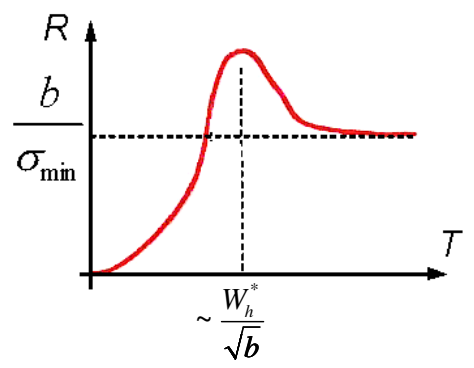

Fig. 4 